\title{
From Spherical Mesopores to Worm-Shaped Mesopores: Morphology Transition in Titania-Polystyrene-b- poly(ethylene oxide) Composite Films with Increasing Sol-Gel Reaction Time
}

\author{
Ya-Jun Cheng, ${ }^{*[a, b]}$ Shaoying Zhou, ${ }^{[b, c]}$ Markus Wolkenhauer, ${ }^{[b]}$ \\ Gina-Gabriela Bumbu, ${ }^{[b]}$ Sebastian Lenz, ${ }^{[b]}$ Mine Memesa, ${ }^{[b]}$ \\ Sebastian Nett, ${ }^{[b]}$ Sebastian Emmerling, ${ }^{[b]}$ Werner Steffen, ${ }^{[b]}$ \\ Stephan V. Roth, ${ }^{[\mathrm{dl}]}$ and Jochen S. Gutmann*[b,e,f]
}

Keywords: Sol-gel processes / Block copolymers / Titania / Thin films / Mesoporous materials

A morphology transition from spherical mesopores to wormshaped mesopores within titania block copolymer composite thin films has been observed by varying the sol-gel reaction time from $40 \mathrm{~min}$ to $48 \mathrm{~h}$ in the four-component templating system of polystyrene- $b$-poly(ethylene oxide) (PS- $b$-PEO), 1,4-dioxane, concentrated $\mathrm{HCl}$, and titanium tetraisopropoxide (TTIP) with a PS- $b$-PEO mass concentration of 0.25 wt.$\%$. The impact of the sol-gel reaction time on the local structure, long-range lateral structure, and vertical structure of the as-prepared, calcined, and UV-degraded thin films as well as the structural changes in solution have been systematically investigated by scanning electron microscopy (SEM), atomic force microscopy (AFM), grazing-incidence small-angle X-ray scattering (GISAXS), X-ray reflectivity (XRR), and dynamic light scattering (DLS). With sol-gel reaction times of up to $5 \mathrm{~h}$, hexagonally organized spherical micelles are present within the as-prepared composite films, in which the core of the spherical micelles is composed of the polystyrene (PS) block, and the corona is composed of the poly(ethylene oxide)-titania (PEO-titania) hybrid. Upon calcination or UV exposure, ordered mesoporous structures are formed owing to the removal of the PS block. With the sol-gel reaction time extended to 25 and $48 \mathrm{~h}$, worm-shaped micelles appear, and their quantity increases with increasing sol-gel reaction time. Worm-shaped mesopores are formed by calcination or UV degradation. The GISAXS results prove that the local structural changes are representative over a macroscopic scale. The XRR results suggest that with the sol-gel reaction time extended to $48 \mathrm{~h}$ there is an additional thin layer beneath the mesoporous titania layer owing to the presence of a large amount of worm-shaped micelles. The results of the DLS studies imply that the morphology transition from spherical micelles to worm-shaped micelles is caused by a fusion process of the spherical micelles in solution.

\section{Introduction}

Nanostructured $\mathrm{TiO}_{2}$ thin films have received considerable research interest in the past decade because of their

[a] Polymers and Composites Division, Ningbo Institute of Materials Technology and Engineering, Chinese Academy of Sciences, 519 Zhuangshi Rd, Zhenhai District, Ningbo, Zhejiang Province 315201, P. R. China

E-mail: chengyj@nimte.ac.cn http://www.nimte.ac.cn/

[b] Max Planck Institute for Polymer Research, Ackermannweg 10, 55128, Mainz, Germany

[c] Department of Chemistry-Biology, University Siegen, 57068 Siegen, Germany

[d] Deutsches Elektronen-Synchrotron (DESY), Notkestr. 85, 22607 Hamburg, Germany

[e] Department of Chemistry and Center for Nanointegration Duisburg-Essen (CENIDE), University of Duisburg-Essen, Campus Essen, 45117 Essen, Germany E-mail: jochen.gutmann@uni-due.de http://www.uni-due.de/

[f] Institute for Physical Chemistry, Johannes Gutenberg University,

Welder Weg 11, 55099 Mainz, Germany wide applications in dye-sensitized solar cells, photocatalysts, gas sensing, and lithium ion batteries. ${ }^{[1]}$ The horizontal and vertical structures of the thin films are essential to the performance of the titania-based devices. In addition to the structure control, the homogeneity of the thin film is also very important, because the performance is a collective contribution over a macroscopic scale, which ranges from at least a few square millimeters to a few square centimeters for the devices at the laboratory phase.

The combination of a polymer templating agent (soft template), sol-gel chemistry, and the evaporation-induced self-assembly (EISA) process has been extensively utilized to synthesize nanostructured $\mathrm{TiO}_{2}$ thin films. ${ }^{[2]}$ In particular, the use of amphiphilic block copolymers such as PEO$b$-PPO- $b$-PEO $[\mathrm{PEO}=$ poly $($ ethylene oxide $), \mathrm{PPO}=$ poly(propylene oxide)], ${ }^{[2 \mathrm{~d}]} \mathrm{PI}-b-\mathrm{PEO}\left(\mathrm{PI}=\right.$ polyisoprene), ${ }^{[2 \mathrm{a}]}$ poly[(ethylene- $c o$-butylene)- $b$-(ethylene oxide)] (PHB- $b$ PEO) ${ }^{[3]}$ PS- $b$-PEO (PS = polystyrene), ${ }^{[4]}$ and PMMA- $b$ PEO $[\text { PMMA }=\text { poly (methyl methacrylate) }]^{[5]}$ as templating 
agents has been intensively explored. Various strategies, including changing the type and composition of the block copolymer, varying the solution constituents and composition, tuning the film deposition and post-treatment conditions, and using the block-polymer aggregates as templates, have been developed to control the morphology of the nanostructured $\mathrm{TiO}_{2}$ multilayer and monolayer thin films. ${ }^{[1 \mathrm{i}, 4 \mathrm{c}, 4 \mathrm{~d}]}$ In addition to these factors, the sol-gel reaction time is also an important factor that influences the morphology of the thin films. However, there have been relatively few studies about the impact of the sol-gel reaction time on the morphology of titania. ${ }^{[6]}$ The sol-gel reaction of the titania precursor [e.g., titanium tetraisopropoxide (TTIP)] in solution is a kinetic process. It continues to change the self-assembly behavior of the titania block copolymer nanostructures by modifying the interactions between the hydrolyzed/condensed titania species and the amphiphilic block copolymer template.

A facile strategy to synthesize nanostructured $\mathrm{TiO}_{2}$ thin films has been developed by our group. ${ }^{[4 a, 5-7]}$ Various morphologies, including nanoparticles, nanovesicles, nanowires, nanogranulas, wormlike aggregates, foams, and flakes, have been synthesized by simply varying the relative mass ratios among PS- $b$-PEO, 1,4-dioxane, concentrated $\mathrm{HCl}$, and titanium tetraisopropoxide (TTIP) in solution. ${ }^{[4 a]}$ In this fourcomponent templating system, PS- $b$-PEO acts as the templating agent, 1,4-dioxane is used as a good solvent for both the PS and PEO blocks, TTIP is used as a precursor for $\mathrm{TiO}_{2}$ and is also a poor solvent for the PS block, and concentrated $\mathrm{HCl}$ is used as a poor solvent for the PS block and a catalyst for the sol-gel process. By systematically varying the composition of the solution constituents, the microphase separation and self-assembly processes of PS- $b$ PEO in solution are tuned. As a result, nanosized titaniaPS- $b$-PEO aggregates with different morphologies are formed, and they can be transferred to a substrate by spin coating to produce nanostructured titania-PS- $b$-PEO composite films.

On the basis of the results of morphology control by variation of the solution composition, the impact of the sol-gel reaction time on the morphology of the titania thin films prepared with 0.50 wt.- $\%$ PS- $b$-PEO was investigated. ${ }^{[6]}$ A morphology evolution from spherical mesopores to worm-shaped mesopores was discovered by simple variation of the sol-gel reaction time from $46 \mathrm{~min}$ to $25 \mathrm{~h}$ with a fixed solution composition and PS- $b$-PEO concentration. Both imaging and scattering studies show that with the solgel reaction time extended to $25 \mathrm{~h}$, a significant amount of worm-shaped domains are formed; this leads to the loss of long-range lateral structure order as demonstrated by grazing-incidence small-angle X-ray scattering (GISAXS). Furthermore, an additional layer of titania is also formed beneath the mesoporous thin film as suggested by X-ray reflectivity (XRR).

As the concentration of PS- $b$-PEO has a significant influence on the morphology of the titania thin films, it is worth investigating the sol-gel reaction time on the morphology change with different PS- $b$-PEO concentrations. As a con- tinuation of our previous research, here we changed the PS$b$-PEO concentration to $0.25 \mathrm{wt} .-\%$ and kept the composition ratio constant (mass ratio: $m_{1,4 \text {-dioxane }} / m_{\mathrm{HCl}} / m_{\mathrm{TTIP}}=$ 0.964:0.007:0.029). ${ }^{[7 \mathrm{~b}]}$ The films were prepared by using the solutions with a series of sol-gel reaction times (40 min, $3,5,25$, and $48 \mathrm{~h}$ ). Atomic force microscopy (AFM) and scanning electron microscopy (SEM) were applied to investigate the local horizontal structures of the thin films. GISAXS was performed to reveal the long-range lateral structures and homogeneity of the nanostructured film. ${ }^{[5]}$ In addition to the local and long-range horizontal structures, the vertical structures normal to the substrate were investigated by XRR. ${ }^{[5,8]}$ The combination of both imaging and scattering techniques provide powerful tools to elucidate the effect of the sol-gel reaction time on the horizontal and vertical structural change of the titania thin films. In addition to the detailed studies on the thin-film nanostructures, the aggregate size change in solution was also studied by dynamic light scattering (DLS). ${ }^{[7 a]}$

\section{Results and Discussion}

Figure 1 shows the high-magnification $\operatorname{SEM}(\mathrm{a} 1, \mathrm{~b} 1, \mathrm{c} 1)$, low-magnification SEM (a2, b2, c2), AFM height (a3, b3, c3), and AFM phase images (a4, b4, c4) of the as-prepared (a1-a4), calcined (b1-b4), and UV-degraded (c1-c4) titania-PS- $b$-PEO composite films prepared with a sol-gel reaction time of $40 \mathrm{~min}$. Figure lal shows spherical dark domains within the as-prepared composite film, which seems like a mesoporous structure. The six-dot fast Fourier transform (FFT) pattern indicates that the spherical domains are organized hexagonally with a characteristic center-to-center distance of $34 \mathrm{~nm}$. Figure 1a2 proves that the spherical dark

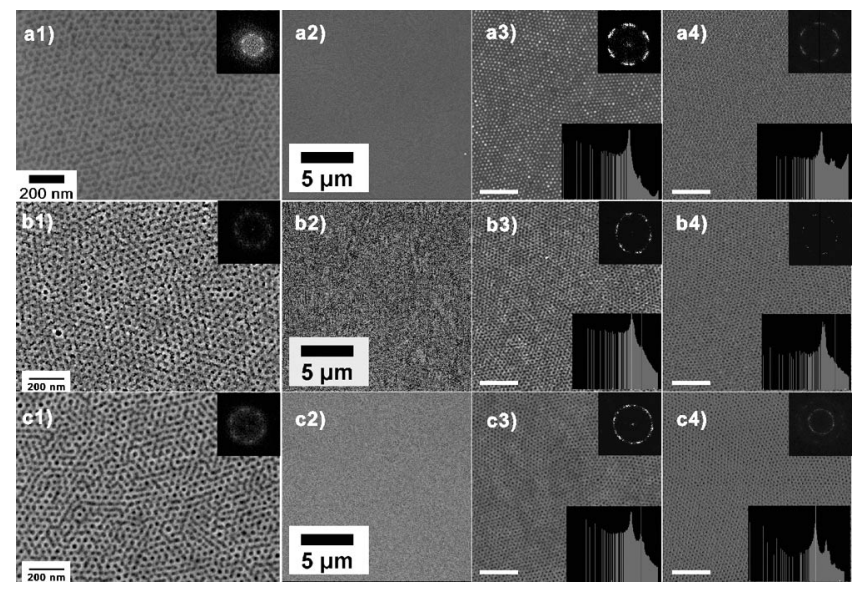

Figure 1. High- (a1, b1, c1) and low-magnification (a2, b2, c2) SEM and AFM height (a3, b3, c3) and phase images (a4, b4, c4) of the titania-block copolymer composite films prepared with a sol-gel reaction time of $40 \mathrm{~min}$ (PS- $b$-PEO concentration: 0.25 wt.\%). (a1-a4) As-prepared; (b1-b4) calcined; (c1-c4) UV-degraded. Height scale of the AFM images: $10 \mathrm{~nm}$, phase scale of the AFM images: $20^{\circ}$. The upper-right insets of the images are the FFT patterns of each image. The lower-bottom insets of the AFM images are the PSD profiles of each image. Scale bar of the AFM images: $400 \mathrm{~nm}$. 
domains are homogeneously distributed over a $\mu \mathrm{m}$-sized area. Interestingly, the seemingly mesoporous feature presented by SEM show dots instead of pores in Figure 1a3, which has a clear mechanical property difference from the matrix according to Figure 1a4. The FFT patterns of Figure $1 \mathrm{a} 3$ and a4 both show six-dot features with center-tocenter distances of $34 \mathrm{~nm}$, which is consistent with Figure 1a1. The power spectral density (PSD) profiles of both Figure 1a3 and a4 show multiple peaks located at 34, 20, 17 , and $13 \mathrm{~nm}$, respectively, corresponding to the ratio $1: 1 /$ $\sqrt{ } 3: 1 / \sqrt{ } 4$. This is in agreement with the theoretical ratio for a hexagonal superstructure and proves that the spherical dark domains are organized with a hexagonal order.

Considering that SEM is based on electron-density contrast, the spherical dark domains in Figure la1 are assumed to be composed of the organic PS block with a lower electron density surrounded by the hybrid PEO-titania continuous network with a higher electron density. ${ }^{[\mathrm{b}]}$ These domains pop out from the surface of the film and exhibit dots in the AFM height image. As the organic PS block has different mechanical properties from the hybrid titaniaPEO network, a good phase contrast is present in the AFM phase image. Regarding the calcined $\mathrm{TiO}_{2}$ thin films, Figure $1 \mathrm{~b} 1$ also shows spherical dark domains, which are homogeneously distributed over $\mu \mathrm{m}$-sized areas according to Figure 1b2. The dotlike FFT pattern of Figure 1b1 indicates that the order of the nanostructures is well retained after calcination. In contrast to that of the as-prepared thin film, the AFM height image shows pores instead of dots (Figure 1b3). This distinct structural difference implies that the spherical domains within the as-prepared composite films are converted to true pores after calcination, whereas the continuous network is retained after calcination. This result confirms the hypothesis that the spherical domain within the as-prepared composite film is composed of the organic PS block and the continuous network is composed of the PEO-titania hybrid. Mesoporous features are also exhibited in the AFM phase image (Figure 1b4) owing to the hardness difference between the pores and the continuous titania network. The FFT patterns of Figure 1b3 and b4 show clear dotlike features with a characteristic center-to-center distance of $33 \mathrm{~nm}$, which is consistent with the value from the as-prepared composite film. Furthermore, the PSD profiles of both Figure 1b3 and b4 show multiple peaks located at 34 and $20 \mathrm{~nm}$, respectively, in agreement with the ratio of $1: 1 / \sqrt{ } 3$. This implies that the mesopores formed after calcination are still organized with hexagonal order. Calcination at high temperature removes the organic template and crystallizes amorphous titania simultaneously, but it tends to promote agglomeration or even collapse of the $\mathrm{TiO}_{2}$ nanostructures under certain conditions. As a compromise, UV exposure has been applied to degrade the organic matrix. The PS block is supposed to be partially removed, and the titania nanostructures remain relatively intact on the substrate. ${ }^{[7 \mathrm{~d}, 9]}$ The composite films degraded by UV light are supposed to possess a better electron-density contrast than the as-prepared films and less structure modification compared to the calcined films. Therefore, it will help to uncover the details of the morphology change within the composite thin films.

The SEM image in Figure 1c1 shows spherical dark domains after UV degradation, and they are homogeneously deposited on the substrate according to Figure 1c2. Unlike Figure 1a3, the AFM height image in Figure 1c3 exhibits pores instead of dots and is similar to Figure 1b3. This implies that the domains composed of the pure PS block are degraded by UV light, and pores remain within the thin film. As the continuous network is composed of the PEOtitania hybrid, it can only be partially degraded by UV light. As a result, the continuous network is retained, and a mesoporous structure is formed. The AFM phase image (Figure 1c4) shows structures consistent with the height image, because there is a distinct mechanical property difference between the pores and the continuous network. The FFT pattern of the SEM image (Figure 1c1) still shows dotlike features with a characteristic distance of $34 \mathrm{~nm}$, indicating that the UV degradation does not deteriorate the order of the nanostructures within the thin films. In particular, the FFT patterns of the AFM height and phase images show both first-order $(34 \mathrm{~nm})$ and second-order $(20 \mathrm{~nm})$ peaks, which indicate that the mesopores are well organized into a hexagonal superstructure after UV exposure. The formation of a hexagonal superstructure is further confirmed by the PSD profiles (Figure 1c3 and c4). Multiple peaks located at 34, 20, 17 and $13 \mathrm{~nm}$ exist and are consistent with the ratio $1: 1 / \sqrt{ } 3: 1 / \sqrt{ } 4: 1 / \sqrt{ } 7$.

When the sol-gel reaction time is increased to $3 \mathrm{~h}$, the as-prepared composite thin film does not exhibit obvious structural changes. Highly ordered spherical domains are surrounded by a continuous network as shown in the high(Figure 2a1) and low-magnification (Figure 2a2) SEM images and AFM height (Figure 2-a3) and phase (Figure $2 \mathrm{a} 4$ ) images. Through calcination, the composite film is converted to mesoporous titania thin film with a good structure order retained, as confirmed by the SEM (Figure $2 \mathrm{~b} 1$ and b2) and AFM images (height image: Figure 2b3; phase image: Figure 2b4). Compared to the as-prepared and calcined thin films, the thin film degraded by UV light also exhibits mesopores with a good structural order, as seen in both the SEM (Figure 2c1 and c2) and AFM images (height image: Figure 2c3; phase image: Figure 2c4). The FFT patterns of the SEM and AFM images of the asprepared, calcined, and UV-degraded thin films still show dotlike features with a characteristic distance of $33 \mathrm{~nm}$. In particular, the FFT patterns of the UV-degraded thin films show not only the first-order peak located at $33 \mathrm{~nm}$ but also a second-order peak located at $20 \mathrm{~nm}$. This implies that the organization of the mesopores is improved after UV exposure. The PSD profiles of the as-prepared and calcined thin films show multiple peaks located at 33, 20, and $17 \mathrm{~nm}$, which indicate that a hexagonal superstructure is present within the as-prepared and calcined thin films. Regarding the UV-degraded thin film, the PSD profiles of both the AFM height and phase images show multiple peaks located at $33,20,17$, and $13 \mathrm{~nm}$. The presence of higher-order peaks further confirms that after UV exposure, the meso- 
pores are organized into a hexagonal superstructure with a better order than that of the as-prepared and calcined thin films.

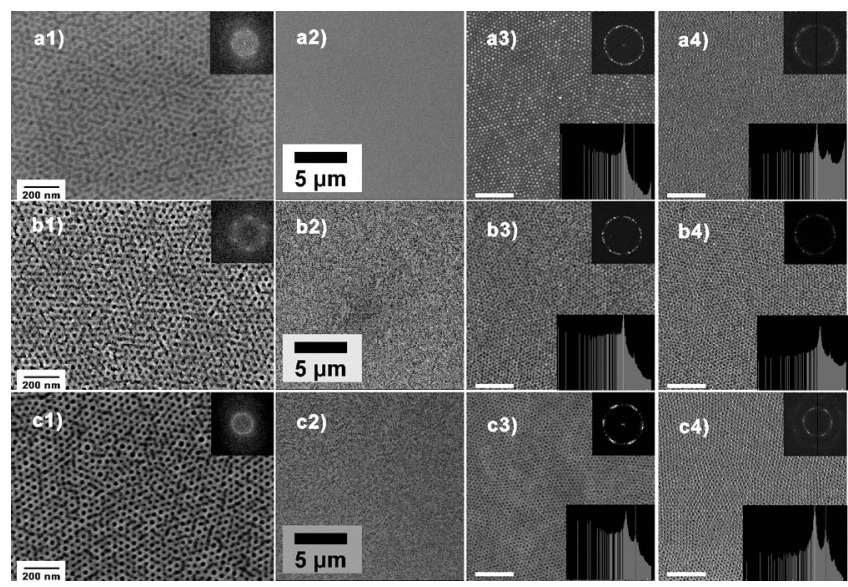

Figure 2. High- (a1, b1, c1) and low-magnification (a2, b2, c2) SEM and AFM height (a3, b3, c3) and phase images (a4, b4, c4) of the titania-block copolymer composite films prepared with a sol-gel reaction time of $3 \mathrm{~h}$ (PS- $b$-PEO concentration: $0.25 \mathrm{wt} .-\%$ ). (a1-a4) As-prepared; (b1-b4) calcined; (c1-c4) UV-degraded. Height scale of the AFM images: $10 \mathrm{~nm}$, phase scale of the AFM images: $20^{\circ}$. The upper-right insets of the images are the FFT patterns of each image. The lower-bottom insets of the AFM images are the PSD profiles of each image. Scale bar of the AFM images: $400 \mathrm{~nm}$.

With the sol-gel reaction time extended to $5 \mathrm{~h}$, the structures of the composite films are still very similar to the films prepared with sol-gel reaction times of $40 \mathrm{~min}$ and $3 \mathrm{~h}$ (Figure 3). Highly ordered spherical domains are present within the as-prepared composite films as shown in the SEM (Figure $3 \mathrm{a} 1$ and a2) and AFM images (height image: Figure 3a3; phase image: Figure 3a4). Mesoporous structures are formed after calcination, as confirmed by the SEM (Figure 3b1 and b2) and AFM images (height image: Figure 3b3; phase image: Figure 3b4). Highly ordered mesopores are also formed after UV treatment as exhibited by the SEM (Figure 3c1 and c2) and AFM images (height image: Figure 3c3; phase image: Figure 3c4). It is worth noting that the mesopores are still organized with high order after calcination or UV exposure as indicated by the ringlike FFT patterns and multiple peaks in the PSD profiles of the images. The characteristic center-to-center distance of the as-prepared composite film is $33 \mathrm{~nm}$ as implied by the FFT patterns of Figure 3a1, a3, and a4. In particular, a second-order peak located at $19 \mathrm{~nm}$ is present in the FFT pattern of the AFM phase image (Figure 3a4).

The PSD profiles of both Figure $3 \mathrm{a} 3$ and a4 show multiple peaks located at 33,19, and $17 \mathrm{~nm}$, which indicate that the spherical domains are organized to form hexagonal superstructure. Concerning the calcined thin film, the FFT patterns of Figure 3b1, b3, and b4 also exhibit a characteristic center-to-center distance of $33 \mathrm{~nm}$. Unlike that of the as-prepared composite film, the FFT pattern of Figure $3 \mathrm{~b} 4$ does not show a second-order peak. However, the PSD profiles (Figure $3 \mathrm{~b} 3$ and b4) still show multiple peaks located

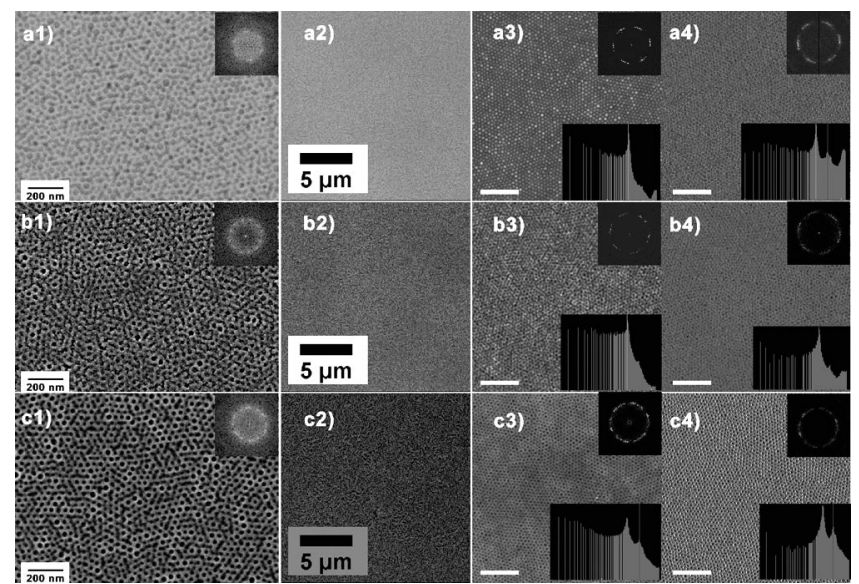

Figure 3. High- (a1, b1, c1) and low-magnification (a2, b2, c2) SEM and AFM height (a3, b3, c3) and phase images (a4, b4, c4) of the titania-block copolymer composite films prepared with a sol-gel reaction time of $5 \mathrm{~h}$ (PS- $b$-PEO concentration: $0.25 \mathrm{wt} .-\%$ ). (a1-a4) As-prepared; (b1-b4) calcined; (c1-c4) UV-degraded. Height scale of the AFM images: $10 \mathrm{~nm}$, phase scale of the AFM images: $20^{\circ}$. The upper-right insets of the images are the FFT patterns of each image. The lower-bottom insets of the AFM images are the PSD profiles of each image. Scale bar: $400 \mathrm{~nm}$.

at 33, 19, and $17 \mathrm{~nm}$. The existence of multiple peaks in the PSD profiles implies that the mesopores are still organized with hexagonal order after calcination. Regarding the thin film treated with UV exposure, the FFT patterns of Figure $3 \mathrm{c} 1, \mathrm{c} 3$, and $\mathrm{c} 4$ imply a characteristic center-to-center distance of $33 \mathrm{~nm}$. Furthermore, an additional second-order peak is present within the FFT pattern of Figure 3c4. Similar to those of the as-prepared and calcined thin films, the PSD profiles of Figure 3c3 and $\mathrm{c} 4$ show multiple peaks located at 33,19, and $17 \mathrm{~nm}$, which indicate that the mesopores formed after UV exposure are organized with hexagonal order.

A significant morphology change occurs when the solgel reaction time is extended to $25 \mathrm{~h}$. In addition to the spherical dark domains, worm-shaped dark domains appear (Figure 4a1). The low-magnification SEM image (Figure 4a2) exhibits areas with different brightness. Further examination of Figure $4 \mathrm{a} 2$ reveals that the dark area corresponds to the worm-shaped domains, and the bright area corresponds to the spherical domains. This is reasonable, because the worm-shaped domains possess larger dark areas than the spherical domains. The AFM height image (Figure 4a3) shows both spherical dots and worm-shaped cylinders; structural details of the worm-shaped cylinders are presented. This shows that some spherical dots are connected with each other to form worm-shaped cylinders, and junctions between the spherical dots are clearly visible. This feature captures the kinetic process of the morphology transition from the spherical dots to the worm-shaped cylinders with increasing sol-gel reaction time. The AFM phase image (Figure 4a4) indicates that the worm-shaped cylinders and spherical dots have similar hardness, different from that of the matrix. The FFT patterns of the SEM and AFM images are smeared out because of the coexistence of the 
spherical and worm-shaped domains. The broad ringlike FFT patterns correspond to a characteristic center-to-center distance of $34 \mathrm{~nm}$. However, the PSD profiles (Figure $4 \mathrm{a} 3$ and a4) still show multiple peaks located at 34, 20, and $17 \mathrm{~nm}$. This implies that the nanostructures are still organized with a hexagonal order despite the coexistence of the spherical and worm-shaped domains.

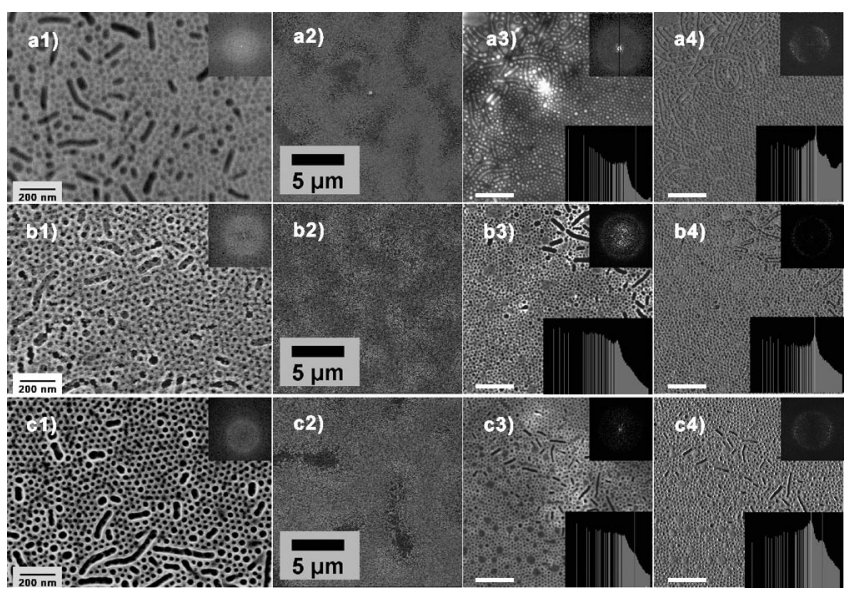

Figure 4. High- (a1, b1, c1) and low-magnification (a2, b2, c2) SEM and AFM height (a3, b3, c3) and phase images (a4, b4, c4) of the titania-block copolymer composite films prepared with a sol-gel reaction time of $25 \mathrm{~h}$ (PS- $b$-PEO concentration: $0.25 \mathrm{wt}$.\%). (a1-a4) As-prepared; (b1-b4) calcined; (c1-c4) UV-degraded. Height scale of the AFM images: $10 \mathrm{~nm}$, phase scale of the AFM images: $20^{\circ}$. The upper-right insets of the images are the FFT patterns of each image. The lower-bottom insets of the AFM images are the PSD profiles of each image. Scale bar of the AFM images: $400 \mathrm{~nm}$.

After calcination, spherical and worm-shaped mesopores form (Figure 4b1). In the low-magnification SEM image, the bright area corresponds to the worm-shaped pores, and the dark area corresponds to the spherical pores. This is likely, because the periphery of the worm-shaped pores protrude from the film surface because of thermal stress during the high-temperature calcination. The AFM height and phase images (height: Figure 4b3; phase: Figure 4b4) show both cylindrical and spherical mesopores. The weak ringlike FFT patterns of Figure 4b1, b3, and b4 indicate a characteristic center-to-center distance of $34 \mathrm{~nm}$. The PSD profiles of both Figure 4b3 and b4 only show one single peak located at $34 \mathrm{~nm}$. This implies that the organization order of the mesopores deteriorates after calcination.

By UV treatment, mesoporous structures are formed as seen in the SEM image Figure 4c1. In the low-magnification SEM image (Figure 4c2), the bright area corresponds to the spherical pores, and the dark area corresponds to the worm-shaped mesopores. This is, because the spherical mesopores possess a smaller pore area than the wormshaped mesopores. This is consistent with the as-prepared composite film and opposite from the calcined film. This difference indicates that the continuous network around the worm-shaped mesopores tends to protrude from the film surface during high-temperature calcination but not in the UV-exposure process. The AFM height image (Figure 4c3) shows cylindrical and spherical mesopores and is consistent with the AFM phase image Figure 4c4. The FFT patterns (Figure 4c1, c3, and c4) correspond to a characteristic center-to-center distance of $34 \mathrm{~nm}$. However, the PSD profiles of the AFM height and phase images still show multiple peaks located at 35, 19, and $16 \mathrm{~nm}$, which implies that the mesopores are organized with a relatively good hexagonal order. With a sol-gel reaction time of less than $5 \mathrm{~h}$, the films prepared with 0.25 wt.- $\%$ PS- $b$-PEO show very similar morphology to the films prepared with 0.50 wt. $\%$ PS- $b-$ PEO. However, with the sol-gel reaction time extended to $25 \mathrm{~h}$, the films with $0.25 \mathrm{wt} .-\%$ PS- $b$-PEO show much less worm-shaped domains than the films with $0.50 \mathrm{wt} .-\%$ PS$b$-PEO. The increase of the PS- $b$-PEO concentration from 0.25 to 0.50 wt. $-\%$ increases the stretching degree of the PS block within the domain. Worm-shaped micelles are preferentially formed to relax the stretching. Furthermore, the increase of PS- $b$-PEO concentration promotes the fusion of the spherical micelles, because more spherical micelles exist and colloid with each other in solution.

With the sol-gel reaction time increased to $48 \mathrm{~h}$, more worm-shaped cylinders exist within the thin film as exhibited by the high-magnification SEM image (Figure 5a1). As a result, the majority of the SEM image in Figure 5a2 is covered by the worm-shaped domains, and the spherical domains are exhibited in the form of patches (bright areas in Figure 5a2). Correspondingly, the AFM height (Figure 5a3) and phase (Figure 5a4) images show more worm-shaped cylinders. Concerning the calcined thin film, the mesoporous structure is still well retained from the SEM images (Figure 5b1 and b2). The AFM height (Figure 5b3) and phase (Figure 5b4) images show that the worm-shaped cylinders are like cracks within the film. After UV degrada-

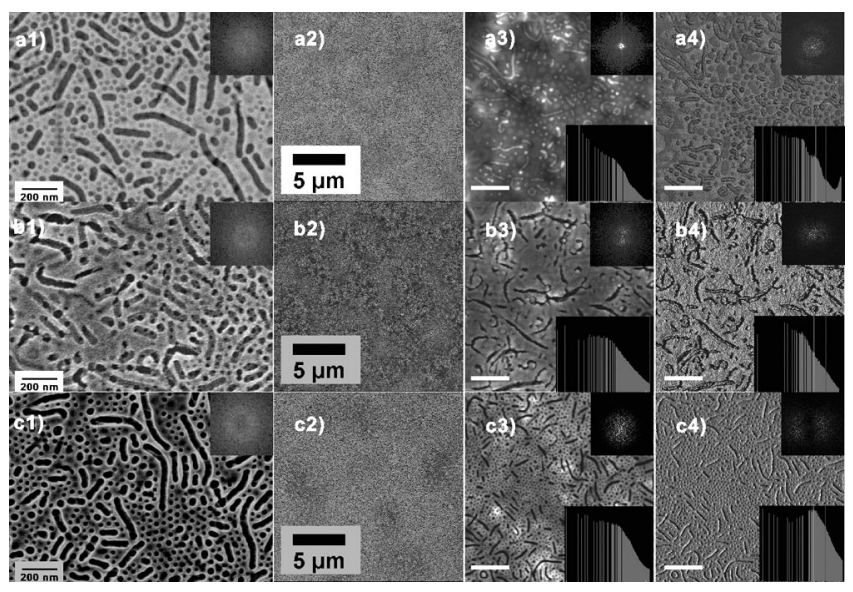

Figure 5. High- (a1, b1, c1) and low-magnification (a2, b2, c2) SEM and AFM height (a3, b3, c3) and phase images (a4, b4, c4) of the titania-block copolymer composite films prepared with a sol-gel reaction time of $48 \mathrm{~h}$ (PS- $b$-PEO concentration: $0.25 \mathrm{wt}$.\%). (a1-a4) As-prepared; (b1-b4) calcined; (c1-c4) UV-degraded. Height scale of the AFM images: $10 \mathrm{~nm}$ (a3, b3), $20 \mathrm{~nm}$ (c3); phase scale of the AFM images: $20^{\circ}$. The upper-right insets of the images are the FFT patterns of each image. The lower-bottom insets of the AFM images are the PSD profiles of each image. Scale bar of the AFM images: $400 \mathrm{~nm}$. 
tion, a mesoporous film is formed according to the SEM images (Figure $5 \mathrm{c} 1$ and $\mathrm{c} 2$ ). The AFM height (Figure 5c3) and phase (Figure 5c4) images clearly show the existence of spherical and worm-shaped pores. Owing to the existence of large amounts of worm-shaped cylinders, the FFT patterns of the SEM and AFM images are smeared out. No distinct peaks can be observed in the PSD profiles of the AFM images.

The morphology transition from spherical domains to worm-shaped domains is supposed to be caused by the fusion process of the spherical micelles in solution. ${ }^{[10]}$ The coronas of the spherical micelles are composed of the PEOtitania hybrids. As the neighboring spherical micelles colloid with each other in solution, the PEO-titania coronas tend to bind together, promoted by the hydrolysis/condensation of TTIP within the micelle coronas. As a result, the spherical micelles fuse together, and new worm-shaped cylindrical micelles form (Scheme 1). ${ }^{[1]}$ This process is visualized in the AFM height images of Figures $4 \mathrm{a} 3$ and 5a3, in which neighboring spherical dots are connected with each other to form worm-shaped cylinders. A similar fusion process of spherical micelles in solution has been reported in the polystyrene- $b$-poly(acrylic acid) (PS- $b$-PAA) system in $N, N$-dimethylformamide (DMF), in which cylinder micelles form through the fusion of spherical micelles. ${ }^{[12]}$ However, the fusion of spherical titania-block copolymer micelles to form cylindrical micelles promoted by a sol-gel reaction has rarely been reported.

The formation of cylindrical micelles leads to an increase of micelle size, which is confirmed by dynamic light scattering (DLS) as shown in Figure 6. The insets show an analysis with the CONTIN algorithm with unweighted re-

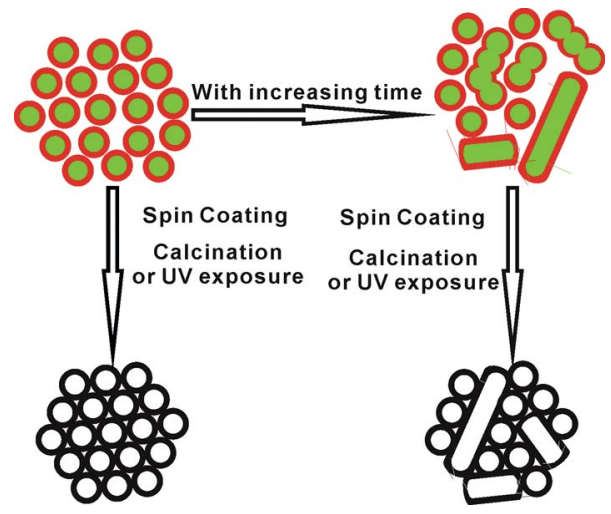

Scheme 1. Formation of worm-shaped domains from spherical domains by a fusion process.

sults for the hydrodynamic radii $R_{\mathrm{h}}$. According to the DLS profiles, within $5 \mathrm{~h}$ the correlation curves and fitted size distribution do not change significantly (Figure 6a-c). Two different structures with individual sizes below 10 and $100 \mathrm{~nm}$ coexist in solution. With the sol-gel reaction time increased to $25 \mathrm{~h}$, the structures with sizes below $10 \mathrm{~nm}$ still exist, but the average size of the larger structures increases (Figure 6d). This trend is also evident when the sol-gel reaction time is further extended to $50 \mathrm{~h}$ (Figure 6e). It seems that the amount of the structures with sizes below $10 \mathrm{~nm}$ significantly decreases, whereas the average size of the larger structures shifts to the range $100-1000 \mathrm{~nm}$, and the amount of the large-sized structures increases dramatically. Although DLS experiments at different angles can give more information about the nanoparticles in solution, the trend of the size change in solution revealed by DLS at a fixed
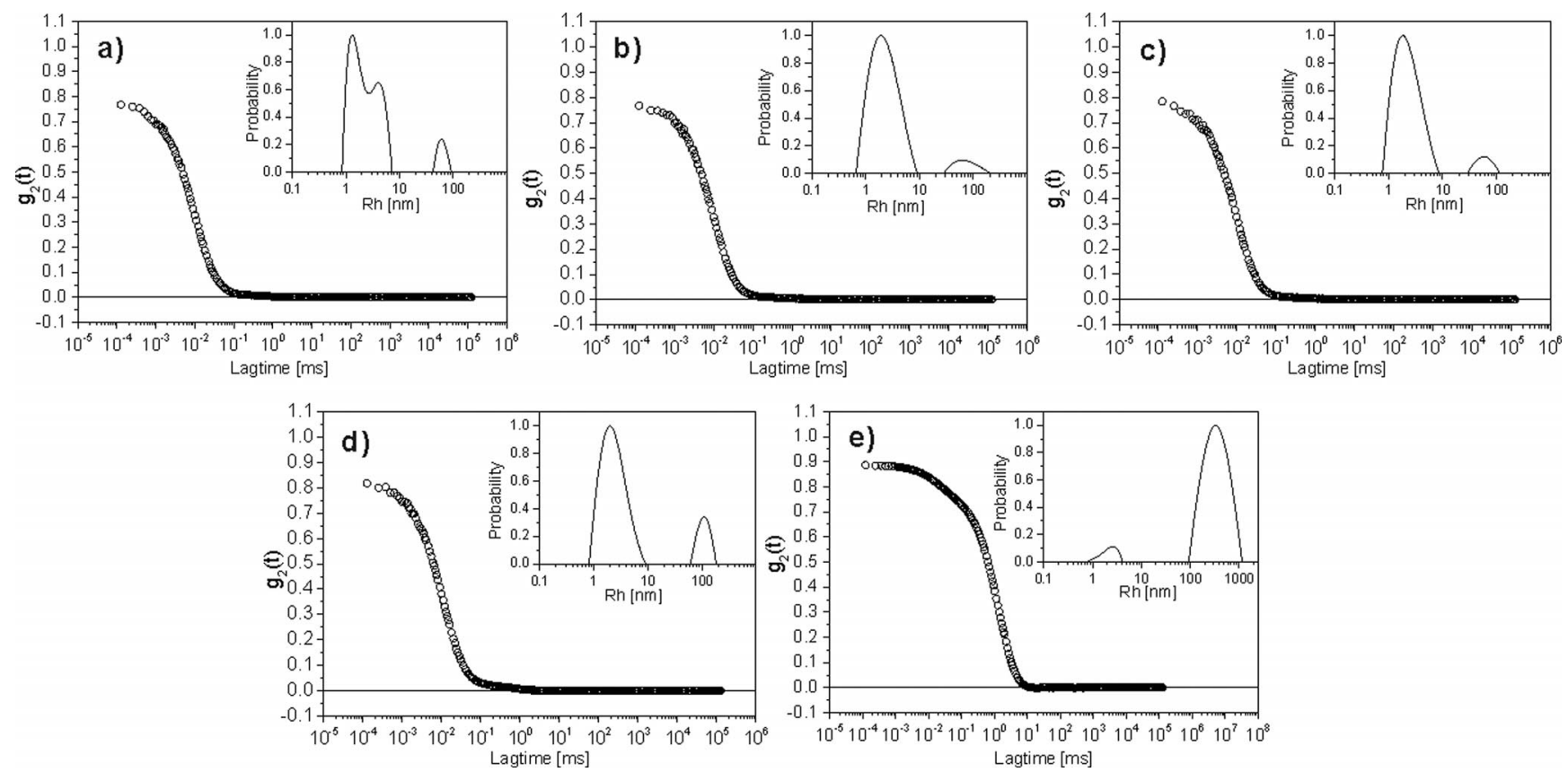

Figure 6. Dynamic light scattering (DLS) profiles of the titania-block copolymer solutions with different sol-gel reaction times (PS- $b$ PEO concentration: 0.25 wt.- $\%$, a: $54 \mathrm{~min}, \mathrm{~b}: 3 \mathrm{~h}, \mathrm{c}: 5 \mathrm{~h}, \mathrm{~d}: 25 \mathrm{~h}$, e: $50 \mathrm{~h}$ ), insets: fitting results (from CONTIN fits, unweighted) of the correlation curves showing the distribution of $R_{\mathrm{h}}$. 
angle already shows consistency with the morphology change within the composite films as shown by AFM and SEM. This implies that the morphology change observed within the composite films is caused by the structure change in solution promoted by increasing sol-gel reaction time.

As imaging techniques such as SEM and AFM can only reveal local structure information of small areas within the thin films, it is very difficult to cover an area of macroscopic length. Furthermore, the homogeneity of the morphology control needs to be effective over a macroscopic scale, which is hard to investigate by SEM and AFM. To obtain statistically relevant information averaged over a macroscopic scale, GISAXS has been used to study the longrange lateral structure of the films. Figure $7 \mathrm{a}-\mathrm{e}$ shows the 2D GISAXS images of the calcined thin films with sol-gel reaction times of $40 \mathrm{~min}, 3,5,25$, and $48 \mathrm{~h}$, respectively. Image $\mathrm{f}$ shows the corresponding out-of-plane (OOP) cuts of the 2D GISAXS images along $q_{y}$. Clear side wing patterns are shown in Figure $7 \mathrm{a}-\mathrm{d}$, and they imply that there is long-range lateral structural order within the film. However, the side wing pattern of Figure $7 \mathrm{e}$ is smeared out, which indicates that the long-range lateral structural order is significantly weakened. The corresponding OOP profiles in Figure $7 f$ prove the trend of the long-range lateral structure change with increasing sol-gel reaction time. With a sol-gel reaction time up to $5 \mathrm{~h}$, the GISAXS profiles show multiple peaks located at 33,19,16, and $12 \mathrm{~nm}$, which indicate a good long-range lateral structural order. The location of the peaks is consistent with the values obtained from the AFM and SEM image analysis. This proves that the spherical domains are organized hexagonally over a macroscopic scale. With a sol-gel reaction time of $25 \mathrm{~h}$, the peaks are broadened and weakened, but the peak positions are still very similar to those of the samples prepared with shorter sol-gel reaction times. This indicates that the hexagonal order of the spherical domains is still retained despite the existence of worm-shaped domains. With the sol-gel reaction time extended to $48 \mathrm{~h}$, the OOP profile only shows two very broad peaks that correspond to ca. 61 and $12 \mathrm{~nm}$. The shift of the peak position to small $q$ values indicates the formation of large superstructures within the composite film. Generally, the change of the OOP profile patterns is consistent with the local structural changes revealed by the AFM and SEM images. This proves that the local structures are representative over a macroscopic scale and the nanostructured thin film is homogeneous over a macroscopic scale.

In addition to the local and long-range lateral structures parallel to the substrate, the vertical structure normal to the substrate is also very important, because it potentially determines the charge-carrier transportation route towards electrodes within thin-film-based devices. Figure 8 shows the corresponding X-ray reflectivity (XRR) profiles of the as-prepared (Figure 8a), calcined (Figure 8b), and UV-degraded thin films (Figure 8c) prepared with sol-gel reaction times of $40 \mathrm{~min}, 3,5,25$, and $48 \mathrm{~h}$. Nice oscillation patterns are present in the as-prepared, calcined, and UV-degraded thin films. From the XRR profiles, it can be seen that the vertical structures do not show big changes with sol-gel
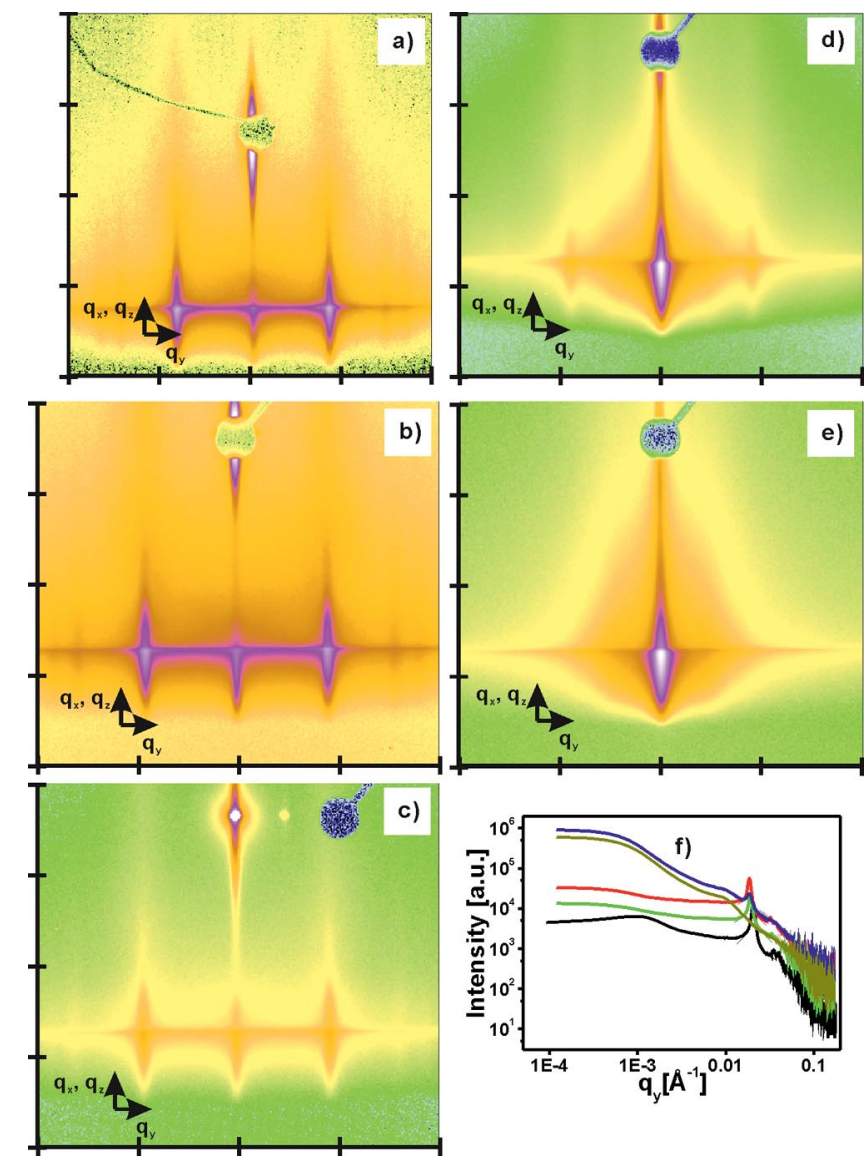

Figure 7. GISAXS profiles of the calcined titania-PS- $b$-PEO composite films prepared with different sol-gel reaction times (PS- $b$ PEO concentration: 0.25 wt. $-\%$ ). (a-e) $2 \mathrm{D}$ image of the GISAXS profiles with a logarithmic scale; (f) OOP profiles of the GISAXS 2D images (a: $40 \mathrm{~min}, \mathrm{~b}: 3 \mathrm{~h}, \mathrm{c:} 5 \mathrm{~h}, \mathrm{~d}: 25 \mathrm{~h}, \mathrm{e}: 48 \mathrm{~h}$ ); black line: $40 \mathrm{~min}$, red line: $3 \mathrm{~h}$, green line: $5 \mathrm{~h}$, blue line: $25 \mathrm{~h}$, dark yellow line: $48 \mathrm{~h} . q_{y}$, range of the 2D GISAXS images: (a) -0.047 to $0.046 \AA^{-1}$, (b-e) -0.041 to $0.041 \AA^{-1}$ (left to right); $q_{z}$ range of the 2D GISAXS images: (a) -0.13 to $0.045 \AA^{-1}$, (b-e) 0.11 to $0.18 \AA^{-1}$ (bottom to top).

reaction times up to $25 \mathrm{~h}$. According to the fitting model, there are monolayers with thicknesses of ca. 50 (as-prepared), 22 (calcined), and $39 \mathrm{~nm}$ (UV-degraded), respectively, which is confirmed by the representative side view SEM image (Figure 8d). However, with a sol-gel reaction time of $48 \mathrm{~h}$, the patterns of the reflectivity profiles change significantly. In addition to the first layer with a similar thickness, an additional thin layer with a thickness of 1$2 \mathrm{~nm}$ with higher scattering-length density exists beneath the first layer. The origin of the second layer is the wormshaped cylinders within the thin films. The hydrophilic $\mathrm{SiO}_{x}$ surface of the $\mathrm{Si}$ substrate has a preferential wetting towards the PEO-titania corona of the micelles. Compared to the spherical micelles, the worm-shaped micelles have a large PEO-titania corona. As a result, patches composed of the titania-PEO hybrid are deposited onto the substrate during spin coating and form an additional layer on the substrate, which is detected by X-ray reflectivity. Concerning the thin films prepared with $0.50 \mathrm{wt} .-\%$ PS- $b$-PEO, the 
second layer appears with a sol-gel reaction of $25 \mathrm{~h}$. However, here the second layer does not appear until the sol-gel reaction time reaches $48 \mathrm{~h}$. This is reasonable, because the amount of the worm-shaped domains in the films prepared with 0.25 wt. $-\%$ PS- $b$-PEO is less than those in the films prepared with 0.50 wt. $-\%$ PS- $b$-PEO.
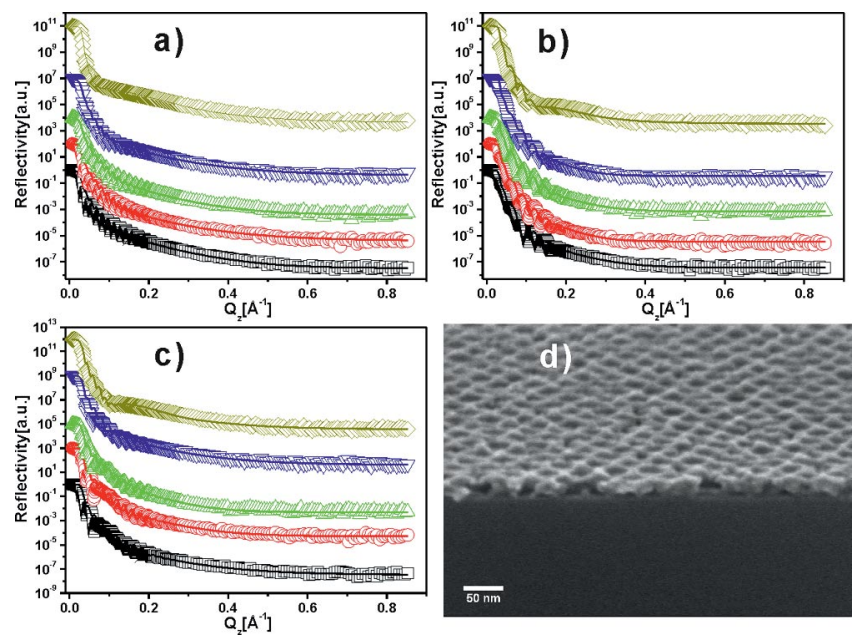

Figure 8. Experimental (symbol) and simulated (line) XRR results of the titania-PS- $b$-PEO composite films prepared with different sol-gel reaction times (black squares: $40 \mathrm{~min}$, red circles: $3 \mathrm{~h}$, green upright triangles: $5 \mathrm{~h}$, blue inverted triangles: $25 \mathrm{~h}$, dark yellow diamonds: $48 \mathrm{~h}$ ) with different treatments. (a) as-prepared; (b) calcined; (c) UV-degraded. (d) Side-view SEM image of the calcined thin film prepared with a sol-gel reaction time of $40 \mathrm{~min}$; (a-c) black squares: $40 \mathrm{~min}$, red circles: $3 \mathrm{~h}$, green upright triangles: $5 \mathrm{~h}$, blue inverted triangles: $25 \mathrm{~h}$.

\section{Conclusions}

A morphology transition from spherical mesopores to worm-shaped mesopores has been achieved by varying the sol-gel reaction time from $40 \mathrm{~min}$ to $48 \mathrm{~h}$ in the four-component templating system of PS- $b$-PEO, 1,4-dioxane, concentrated $\mathrm{HCl}$, and TTIP with a PS- $b$-PEO mass concentration of $0.25 \mathrm{wt} .-\%$. The impact of the sol-gel reaction time on the local structure, long-range lateral structure, and vertical structure of the as-prepared, calcined, and UV-degraded composite films has been systematically investigated by AFM, SEM, X-ray reflectivity, and GISAXS. The corresponding structural changes in solution have been studied by DLS. With a sol-gel reaction time up to $5 \mathrm{~h}$, only spherical domains are formed, and they are organized hexagonally within the films. Mesoporous structures are formed after calcination or UV exposure, and structural order is still well retained after treatment. When the sol-gel reaction time is extended to $25 \mathrm{~h}$, in addition to spherical domains, worm-shaped domains appear, likely because of the fusion of neighboring spherical domains. However, the films still possess local and long-range lateral structural order as suggested by AFM, SEM, and GISAXS. With the sol-gel reaction time further increased to $48 \mathrm{~h}$, large amounts of wormshaped domains are present. The X-ray reflectivity results show that the films are monolayer in nature with sol-gel reaction times up to $25 \mathrm{~h}$. With the sol-gel reaction time extended to $48 \mathrm{~h}$, an additional thin layer of titania exists owing to the preferential wetting of the titania-PEO corona of the worm-shaped micelles on the $\mathrm{SiO}_{x}$ substrate.

\section{Experimental Section}

An asymmetric diblock copolymer of polystyrene-block-poly(ethylene oxide) (PS- $b$-PEO) with a number average molecular weight of 19000 for PS and 6400 for PEO [polydispersity index (PDI): 1.05] was purchased from Polymer Source Inc. Titanium tetraisopropoxide (TTIP, 97\%) was purchased from Sigma-Aldrich. Analyticalgrade 1,4-dioxane and concentrated $\mathrm{HCl}(\min .37 \%$ ) were received from Riedel-de Haën.

Sample solutions were prepared according to the following procedure. PS-b-PEO (0.01 g) was mixed with 1,4-dioxane (ca. $4.0 \mathrm{~g})$, $37 \% \mathrm{HCl}(0.03 \mathrm{~g})$, and TTIP $(0.12 \mathrm{~g})$ within $6 \mathrm{~min}$. The stock solutions were stirred for $40 \mathrm{~min}, 3,5,25$, and $48 \mathrm{~h}$ before spin coating.

Films were prepared on $\mathrm{Si}(100)$ substrates by spin coating for $60 \mathrm{~s}$ with a Süss MicroTec Delta 80 spin coater under ambient conditions (temperature: $21^{\circ} \mathrm{C}$, relative humidity: $55-65 \%$, rotation speed: $2000 \mathrm{rpm}$, acceleration speed: $2000 \mathrm{rpm} / \mathrm{s}$, lid open).

Calcination of the composite thin films was performed at $400{ }^{\circ} \mathrm{C}$ for $4 \mathrm{~h}$ in air with a ramp rate of $6.25^{\circ} \mathrm{C} / \mathrm{min}$ starting from room temperature. After calcination, the samples were cooled to room temperature in the furnace.

UV degradation was conducted with UV light of $254 \mathrm{~nm}$ major wavelength with a fluence of $25 \mathrm{~J} / \mathrm{cm}^{2}$ (XX-15S, UVP, Inc.) at room temperature for $27 \mathrm{~h}$ in air to degrade the block copolymer within the thin films.

AFM images were recorded with a Digital Instruments Dimension $^{\text {TM }} 3100$ scanning force microscope in tapping mode equipped with Olympus cantilevers (spring constant ranging between 33.2 and $65.7 \mathrm{~N} / \mathrm{m}$ and resonant a frequency of $277.3-346.3 \mathrm{kHz}$ ). The images were analyzed by using the Nanoscope $5.12 \mathrm{r} 5$ software.

SEM images were obtained with a field emission SEM (LEO 1530 "Gemini"). The accelerating voltage was $1 \mathrm{kV}$. FFT patterns were obtained by using the ImageJ $1.33 \mathrm{U}$ software, and areas of $512 \times 512$ pixels of the SEM images were randomly selected for analysis.

XRR experiments were conducted with a surface XRD-TT3003 diffractometer (Seifert, Germany) equipped with a Göbel mirror, wavelength $1.542 \AA$. The modeling of the experimental profiles was conducted by the Parratt 32 software, version 1.5 provided by the Berlin Neutron Scattering Center at the Hahn-Meitner Institute.

GISAXS measurements were performed at the BW4 beamline of the DORIS III storage ring at HASYLAB/DESY by using a completely evacuated sample-to-detector pathway (2.00 or $2.20 \mathrm{~m}$ ) and a wavelength of $\lambda=1.38 \AA$. The scattering images were analyzed in terms of out-of-plane (OOP) scans along the $q_{y}$ axis of the scattering images at $q_{z}$ values corresponding to the critical angle of $\mathrm{TiO}_{2}$. For such cuts, the transmission functions of the incident and scattered waves only entered as constant scaling factors and do not depend on the precise functional shape of the transmission functions. In this way, the OOP scans were selective to the scattering information of a particular material, that is, at a given scatteringlength density, as they took advantage of the intensity increase of the transmission function at the critical angle. 
Dynamic light scattering was performed with a custom-built goniometer setup with a Verdi V2 laser (Coherent, wavelength $532 \mathrm{~nm}$ ). The incident light of a maximum power of $100 \mathrm{~mW}$ on the sample was vertically polarized with respect to the scattering plane by a Glan prism with an extinction ratio of $10^{-5}$. Of the scattered light, only the vertically polarized component selected by a GlanThompson prism with an extinction ratio of $10^{-8}$ was analyzed. Single-mode fiber detection was utilized with a beam splitter and two avalanche diodes (Perkin-Elmer) as detectors. The autocorrelator (ALV-6010/160 high speed multiple tau digital Korrelator; ALV, Langen, Germany) was run in pseudo-cross-correlation to eliminate correlated noise from the detectors at short times. All samples were filtered into dust-free light-scattering cells through a $0.2 \mu \mathrm{m}$ Millipore Teflon filter to remove dust.

\section{Acknowledgments}

The authors would like to thank Gunnar Glasser for help with FESEM and the provision of synchrotron beam time from BW4 at DESY. The financial support from the Max Planck Society is greatly appreciated.

[1] a) P. Innocenzi, L. Malfatti, Chem. Soc. Rev. 2013, 42, 4198 4216; b) M. Anaya, M. E. Calvo, J. M. Luque-Raigon, H. Miguez, J. Am. Chem. Soc. 2013, 135, 7803-7806; c) A. G. Dylla, G. Henkelman, K. J. Stevenson, Acc. Chem. Res. 2013, 46, 1104-1112; d) D. H. Chen, R. A. Caruso, Adv. Funct. Mater. 2013, 23, 1356-1374; e) M. Munz, M. T. Langridge, K. K. Devarepally, D. C. Cox, P. Patel, N. A. Martin, G. Vargha, V. Stolojan, S. White, R. J. Curry, ACS Appl. Mater. Interfaces 2013, 5, 1197-1205; f) X. Chen, S. S. Mao, Chem. Rev. 2007, 107, 2891-2959; g) D. W. Manley, R. T. McBurney, P. Miller, R. F. Howe, S. Rhydderch, J. C. Walton, J. Am. Chem. Soc. 2012, 134, 13580-13583; h) A. Hagfeldt, G. Boschloo, L. C. Sun, L. Kloo, H. Pettersson, Chem. Rev. 2010, 110, 6595-6663; i) J. H. Pan, X. S. Zhao, W. I. Lee, Chem. Eng. J. 2011, 170, 363-380.

[2] a) M. C. Orilall, U. Wiesner, Chem. Soc. Rev. 2011, 40, 520535; b) D. Grosso, F. Ribot, C. Boissiere, C. Sanchez, Chem. Soc. Rev. 2011, 40, 829-848; c) D. Grosso, C. Boissiere, B. Smarsly, T. Brezesinski, N. Pinna, P. A. Albouy, H. Amenitsch,
M. Antonietti, C. Sanchez, Nat. Mater. 2004, 3, 787-792; d) G. Soler-Illia, E. L. Crepaldi, D. Grosso, C. Sanchez, Curr. Opin. Colloid Interface Sci. 2003, 8, 109-126; e) E. L. Crepaldi, G. Soler-Illia, D. Grosso, F. Cagnol, F. Ribot, C. Sanchez, J. Am. Chem. Soc. 2003, 125, 9770-9786; f) G. J. D. Soler-Illia, C. Sanchez, B. Lebeau, J. Patarin, Chem. Rev. 2002, 102, 4093-4138.

[3] T. Brezesinski, M. Groenewolt, A. Gibaud, N. Pinna, M. Antonietti, B. M. Smarsly, Adv. Mater. 2006, 18, 2260-2263.

[4] a) Y. J. Cheng, J. S. Gutmann, J. Am. Chem. Soc. 2006, 128, 4658-4674; b) Y. H. Deng, J. Wei, Z. K. Sun, D. Y. Zhao, Chem. Soc. Rev. 2013, 42, 4054 4070; c) D. Chandra, M. Bekki, M. Nakamura, S. Sonezaki, T. Ohji, K. Kato, T. Kimura, J. Mater. Chem. 2011, 21, 5738-5744; d) D. Chandra, T. Ohji, K. Kato, T. Kimura, Phys. Chem. Chem. Phys. 2011, 13, 12529-12535; e) T. Kimura, Chem. Asian J. 2011, 6, 3236-3242; f) T. Kimura, Macromol. Rapid Commun. 2013, 34, 423-430; g) T. Kimura, Y. Yamauchi, Langmuir 2012, 28, 12901-12908; h) X. Li, J. Peng, J. H. Kang, J. H. Choy, M. Steinhart, W. Knoll, D. H. Kim, Soft Matter 2008, 4, 515-521; i) B. Ma, J. Ma, G. K. L. Goh, J. Mater. Sci. 2008, 43, 4297-4302.

[5] Y. J. Cheng, P. Muller-Buschbaum, J. S. Gutmann, Small 2007, 3, 1379-1382.

[6] Y. J. Cheng, S. Y. Zhou, M. Wolkenhauer, G. G. Bumbu, S. Lenz, M. Memesa, S. Nett, S. Emmerling, W. Steffen, J. S. Gutmann, Eur. J. Inorg. Chem. 2013, 1127-1133.

[7] a) Y. J. Cheng, L. Zhi, W. Steffen, J. S. Gutmann, Chem. Mater. 2008, 20, 6580-6582; b) Y. J. Cheng, S. Y. Zhou, J. S. Gutmann, Macromol. Rapid Commun. 2007, 28, 1392-1396; c) M. Z. Yin, Y. J. Cheng, M. Y. Liu, J. S. Gutmann, K. Mullen, Angew. Chem. 2008, 120, 8528; Angew. Chem. Int. Ed. 2008, 47, 8400 8403; d) Y. J. Cheng, M. Wolkenhauer, G. G. Bumbu, J. S. Gutmann, Macromol. Rapid Commun. 2012, 33, 218-224.

[8] M. J. Henderson, A. Gibaud, J. F. Bardeau, J. W. White, J. Mater. Chem. 2006, 16, 2478-2484.

[9] D. H. Kim, Z. C. Sun, T. P. Russell, W. Knoll, J. S. Gutmann, Adv. Funct. Mater. 2005, 15, 1160-1164.

[10] Y. Y. Mai, A. Eisenberg, Macromolecules 2011, 44, 3179-3183.

[11] D. Scalarone, J. Tata, F. Caldera, M. Lazzari, O. Chiantore, Mater. Chem. Phys. 2011, 128, 166-171.

[12] P. L. Soo, A. Eisenberg, J. Polym. Sci., Part B: Polym. Phys. 2004, 42, 923-938.

Received: September 25, 2013 Published Online: 


\section{Thin Films}

Y.-J. Cheng,* S. Zhou, M. Wolkenhauer, G.-G. Bumbu, S. Lenz, M. Memesa, S. Nett, S. Emmerling, W. Steffen, S. V. Roth, J. S. Gutmann* 1-10

From Spherical Mesopores to WormShaped Mesopores: Morphology Transition in Titania-Polystyrene- $b$-poly(ethylene oxide) Composite Films with Increasing Sol-Gel Reaction Time

Keywords: Sol-gel processes / Block copolymers / Titania / Thin films / Mesoporous materials

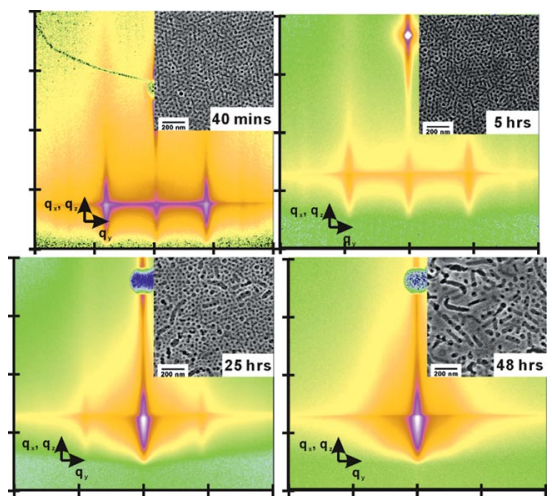

A morphology transition from spherical mesopores to worm-shaped mesopores within titania-polystyrene- $b$-poly(ethylene oxide) (titania-PS- $b$-PEO) composite thin films is achieved by varying the sol-gel reaction time from $40 \mathrm{~min}$ to $48 \mathrm{~h}$ with 0.25 wt. $-\%$ PS- $b$-PEO and fixed solution composition, as confirmed by imaging and scattering techniques. 